${ }^{1}$ MSc. Especialista en
Reumatología y Medicina Reumatología y Medicina Satisfactoria.Investigador a g r e g a d o. Doc e n t e Investigador Universidad Nacional de Chimborazo, Docente Escuela Superior Politécnica de Chimborazo. Hospital Andino Riobamba, Chimborazo, Ecuador.

2Estudiante del Noveno semestre de la Carrera de Medicina, Presidente de la Asociación Científica de Estudiantes de Medicina de la Universidad Naciona de Chimborazo, Latacunga, Cotopaxi.

Correspondencia a:

Nombre: Dr. Urbano Solis Cartas, MSc.

Correo: umsmwork74@gmail. com.

Telefono: +593969467803. ORCID: https://orcid.org/0000 0003-0350-6333

https://orcid.org/0000-0003 1553-4606

\title{
EL SÍNDROME DE SJÖGREN Y SU INFLUENCIA EN LA CALIDAD DE VIDA
}

\author{
SJÖGREN'S SYNDROME AND ITS INFLUENCE ON QUALITY OF LIFE
}

\author{
Mg. Dr. Urbano Solis Cartas ${ }^{1}$, Joel Alejandro Barreto Araujo
}

\section{RESUMEN}

Introducción: Las afecciones reumáticas constituyen un grupo de enfermedades que además de afectar el aparato locomotor, también afectan otros órganos y sistemas, lo que genera discapacidad funcional y disminución de la percepción de calidad de vida; dentro de estas afecciones se incluye el síndrome de Sjögren. Objetivo: Determinar la percepción en la calidad de vida en pacientes con síndrome de Sjögren. Metodología: Estudio descriptivo, retrospectivo y con enfoque mixto; universo de 457 pacientes con diagnóstico de síndrome de Sjögren. Muestra constituida por 417 pacientes, se aplicó el cuestionario de láminas Coop/Wonca para determinar la percepción de calidad de vida y la versión cubana del Health Assessment Questionnaire para determinar capacidad funcional, a la vez también se utilizó el coeficiente de correlación de Pearson para determinar asociación entre las variables del estudio. Resultados: La puntuación de percepción en la calidad de vida global fue de $21,78 \%$ y las dimensiones más afectadas fueron el dolor, las actividades sociales y cotidianas; siendo diferentes entre ambos sexos. Conclusión: Existió correlación positiva notoria entre la presencia de discapacidad funcional y las puntuaciones de calidad de vida relacionada con la salud; mientras mayor fue las puntuaciones de discapacidad peor fue la percepción de calidad de vida de los pacientes.

\section{ABSTRACT}

Introduction: Rheumatic affections constitute a group of diseases that, in addition to affecting the locomotor system, also affect other organs and organ systems, which generates functional disability and a decrease in the perception of quality of life; these conditions include Sjögren's syndrome. Materials and methods. Descriptive, retrospective study with a mixed approach; universe of 457 patients diagnosed with Sjögren's syndrome. Sample made up of 417 patients, the Coop / Wonca slide questionnaire was applied to determine the perception of quality

Palabras clave: Calidad de vida enfermedades autoinmunes, enfermedades reumáticas, evaluación de la discapacidad, síndrome de Sjögren.

Keywords: Quality of life, autoimmune diseases, rheumatic diseases, evaluation of disability, Sjögren syndrome.

Procedencia y arbitraje: no comisionado, sometido a arbitraje externo.

Recibido para publicación 20 de abril de 2020 Aceptado para publicación 13 de junio de 2021

Citar como:

Solis Cartas U, Barreto Araujo JA. EL SÍNDROME DE SJÖGREN Y SU INFLUENCIA EN LA CALIDAD DE VIDA. Rev Cient Cienc Med 2021;24(1): 144-150

of life and the Cuban version of the Health Assessment Questionnaire to determine functional capacity. Pearson's correlation coefficient was used to determine association between the study variables. Results: The perception score in the global quality of life was $21.78 \%$ and the most affected dimensions were pain, social and daily activities; being different between both sexes. Conclusions: there was a strong positive correlation between the presence of functional disability and health-related quality of life scores; the higher the disability scores, the worse the perception of quality of life of the patients.

\section{INTRODUCCIÓN}

El síndrome de Sjögren (SS) es una Eenfermedad autoinmune, crónica cuya base inflamatoria genera sustitución del parénquima glandular por tejido conectivo'. Es reportada como una enfermedad de distribución universal que afecta a ambos sexos, pero con predominio por el sexo femenino; suele presentarse con mayor frecuencia por encima de los 40 años con un pico de incidencia a partir de los 55 años de edad ${ }^{2,3}$. Se reporta una prevalencia entre el 0,09\% y el 2,7\% de la población mundial ${ }^{4}$. En Europa se estima una prevalencia de 1,8 por cada 1000 habitantes, mientras que en Estados Unidos la cifra alcanza a 2,5 personas por cada 1000 habitantes $^{5}$.Es considerada una enfermedad multifactorial que engloba distintos factores capaces de generar las alteraciones inmunológicas. Dentro de estos factores se señalan infecciones virales, sustancias químicas, radiaciones, trastornos inmunológicos previos, componente genético y presencia de otras enfermedades entre otros ${ }^{1}$, 4,6 .

El SS se clasifica en primario o secundario a otras enfermedades. Se plantea que se asocia al $30 \%$ de los pacientes con artritis reumatoide, $20 \%$ de casos con esclerosis sistémica y $10 \%$ de pacientes con lupus eritematosos sistémico ${ }^{5,6}$. También ha sido reportado en el $16 \%$ a los pacientes con diabetes mellitus y en el $13 \%$ de ca- 
sos con diagnóstico de hipotiroidismo ${ }^{6,7}$.

Las principales manifestaciones clínicas del SS incluyen la presencia de un cuadro inflamatorio poliarticular y la afectación sistémica con predominio de sequedad de mucosas. Las complicaciones afectan cualquier sistema de órganos del cuerpo humano y serian responsables sobre la aparición de discapacidad funcional y afectación de la percepción en la calidad de vida relacionada con la salud $(\mathrm{CVRS})^{7}$. En Ecuador no existen estudios orientados a la identificación de la percepción de CVRS en pacientes con SS, lo que motiva la realización de esta investigación.

Es por ello que teniendo en cuenta la relativa frecuencia con que se presenta el SS, su repercusión de la enfermedad en la capacidad funcional y la percepción de CVRS de los pacientes y la no existencia de estudios relacionados con este tema en Ecuador; se decide realizar esta investigación con el objetivo de determinar la percepción de CVRS de los pacientes con diagnóstico de SS.

\section{MATERIALES Y MÉTODOS}

Se realizó una investigación básica, diseño descriptivo, retrospectivo, de enfoque mixto. El universo estuvo conformado por 457 pacientes con diagnóstico de SS que acudieron al servicio de consulta externa del Hospital Andino de Riobamba, durante el periodo comprendido entre enero del 2008 y diciembre del 2018. La muestra quedó conformada por 417 pacientes que debieron cumplir los criterios de inclusión definidos para el estudio.

Se incluyeron, hombres y mujeres con diagnóstico de SS mayores de 20 años que aceptaron participar en el estudio, se excluyeron a los pacientes con deterioro cognitivo y se eliminaron los cuestionarios incompletos.

Para realizar el analisis de la muestra se utilizó la"fórmula de cálculo muestra para poblaciones conocidas"8; después de identificar el tamaño de la muestra se procedió a confeccionarla con la utilización de método aleatorio simple ${ }^{8}$.

Durante el desarrollo de la investigación se utilizaron tres variables de investigación: Características sociodemográficas que incluyó las dimensiones edad, sexo, tiempo de evolución, tipo de SS y tipo de enfermedad de base; percepción de CVRS y capacidad funcional.
Se utilizaron tres instrumentos para recolectar la información; el primero de ellos fue una encuesta diseñada específicamente para el estudio, que fue sometida a la valoración de expertos que emitieron criterios positivos sobre la misma que constaba con un total de 8 preguntas. Se utilizó el cuestionario de láminas Coop/Wonca para determinar la percepción de CVRS y la versión cubana del Health Assessment Questionnaire (HAQ-CU) para determinar capacidad funcional ${ }^{9,10}$.

Las láminas Coop/Wonca son un formulario genérico, validado en español, de amplia utilización que integra elementos visuales. Evalúa 7 dimensiones y en cada una existen posibilidades de respuesta con una puntuación que va desde 1 a 5 puntos, siendo la puntuación mayor un indicador de peor percepción de CVRS $^{11,12}$.

El cuestionario $\mathrm{HAQ}-\mathrm{CU}$ es una versión validada en Cuba que ha sido utilizada para determinar capacidad funcional de pacientes con enfermedades reumáticas, se basa en preguntar a los pacientes sobre su capacidad para realizar distintas actividades de la vida diaria; abarca 8 ítems y las puntuaciones van desde 0 puntos (totalmente capaz de realizar la actividad), hasta 3 puntos (totalmente dependiente para realizar la actividad) ${ }^{12,13}$.

Se establece que puntuaciones por debajo de 0,5 puntos evidencian que no existe discapacidad funcional, puntuaciones entre 0,5 y 1 punto muestran discapacidad ligera, puntuaciones mayores de 1 punto y menores de 2 puntos son representativo de discapacidad moderada y los pacientes que obtengan puntuación igual o superior a 2 puntos son considerados con discapacidad severa ${ }^{13}$.

La información fue procesada de forma automatizada mediante la utilización del paquete estadístico SPSS en su versión 20,5 para Windows; se determinaron frecuencias absolutas, porcentajes para las variables cualitativas, medidas de tendencia central y de dispersión. Se utilizó el coeficiente de correlación Pearson para determinar la asociación entre CVRS y capacidad funcional. El nivel de confianza fue determinado en el $95 \%$ con un margen de error del $5 \%$ y una significación estadística fijada en una $p=0,05$.

Se tuvieron en cuenta los elementos estipulados en la declaración de Helsinki II; todos los pacientes fueron informados de los 
objetivos, metodología del estudio, siendo incorporados a la investigación después de firmar el consentimiento informado; los datos obtenidos fueron tratados bajo total confidencialidad y con fines investigativos. La participación en el estudio fue voluntaria y sin costo alguno.

\section{RESULTADOS}

En la tabla 1 se muestra un promedio de edad de 53,22 años (DE 15,8 años); el grupo de edad más prevalente fue el que incluyó pacientes entre 40 y 60 años (frecuencia 266; $63,79 \%$ y $p=0,04)$. Existió un predominio del sexo femenino (368 casos y $88,25 \%$ ) que fue estadísticamente significativo, al igual que el tiempo de evolución de la enfermedad, del cual el rango que mayor representatividad alcanzó fue el comprendido entre 3 y 5 años (271 pacientes, 64,99\%) con una $p=0,041$.

También se analizó el tipo de SS en los pacientes; en 354 casos se pudo comprobar el origen secundario de la enfermedad $(85,89 \%)$, representado por una $p=0,01$, siendo estadísticamente significativo. Las enfermedades que mayor representatividad tuvieron dentro de los pacientes con SS secundario, fueron la artritis reumatoide (AR) (175 pacientes; $49,43 \%$ y $p=0,047)$, el hipotiroidismo (73 casos; 20,62\% y $\mathrm{p}=0,08$ ), la diabetes mellitus (51 pacientes; $14,40 \%$ y $\mathrm{p}=0,095)$ y el lupus eritematoso sistémico $(13,28 \%)$.

El análisis de la percepción de CVRS se muestra en la tabla 2. La puntuación global obtenida en el estudio fue de 22,78 con una DE de 7,22 en el caso de los pacientes masculinos la media fue de 18,73 y en las féminas de 22,43. Las dimensiones que de forma general mayor afectación presentaron fueron la dimensión dolor $(4,20$ y DE 0,80$)$, actividades sociales (3.99 y $D E 1,01)$ y la relacionada con las actividades cotidianas (3,92 y DE 1,08).

Al analizar la capacidad funcional de los pacientes (tabla 3 ) se obtuvo que el $61,87 \%$ de los pacientes con SS presentaron algún tipo de discapacidad funcional. Dentro de los pacientes que se identificaron con discapacidad funcional destacaron, como dato estadísticamente significativo el $51,55 \%$ que presentaron discapacidad moderada.

En la tabla 4 se muestra la distribución de pacientes con SS en torno a variables sociodemográficas y percepción de CVRS. Se observa que al analizar el tiempo de evolución de la enfermedad la peor percepción de CVRS la mostraron los pacientes con más de 5 años de diagnosticado el SS (media de 24,03 y DE 4,97).

Al analizar el resultado de la correlación de Pearson entre la presencia de discapacidad funcional y las puntuaciones de percepción de CVRS se encontró una correlación positiva fuerte entre estas dos variables $(0,79)$.

\section{DISCUSIÓN}

En el presente estudio se encontró un promedio de edad de 53,22 años con predominio de pacientes entre 40 y 60 años. Este resultado es similar al reportado por otras investigaciones en las cuales se plantea que el SS presenta un predominio de afectación en pacientes por encima de 40 años de edad ${ }^{2,3}$. Una posible explicación puede estar basada en dos elementos fundamentales: el primero de ellos se relaciona con las alteraciones hormonales que ocurren en el sexo femenino a partir de los 40 años, donde existe un cese brusco de la función ovárica con disminución de la producción de estrógenos ${ }^{12,}$ el segundo elemento se relaciona con el aumento de la frecuencia de enfermedades reumáticas y endocrino metabólicas, que son las principales afecciones que podrían generar secundariamente la aparición del SS ${ }^{14,15}$.

Existió un predominio de pacientes con SS secundario; es importante señalar que otras investigaciones también reportan que la AR es la enfermedad reumática que mayormente da origen al $\mathrm{SS}^{6}$. En el caso de la elevada prevalencia del hipotiroidismo es importante destacar, que aunque no existen reportes en la literatura, si se observa en la práctica médica diaria una mayor frecuencia de presentación de la enfermedad en el contexto de la investigación.

Al analizar la puntuación de percepción de CVRS se observa un valor superior a 22 puntos. En el estudio de Fernández Martínez y otros, se estudiaron pacientes con SS primario y se concluyó que la enfermedad provoca un importante daño en la cavidad oral lo que genera una disminución considerable de la calidad de vida ${ }^{16}$.

La dimensión más afectada en ambos sexos fue la del dolor, el cual es predominante en pacientesconSS porencimadelas características inflamatorias ${ }^{17}$. Otras dimensiones que también 
Tabla 1. Distribución de pacientes según características sociodemográficas.

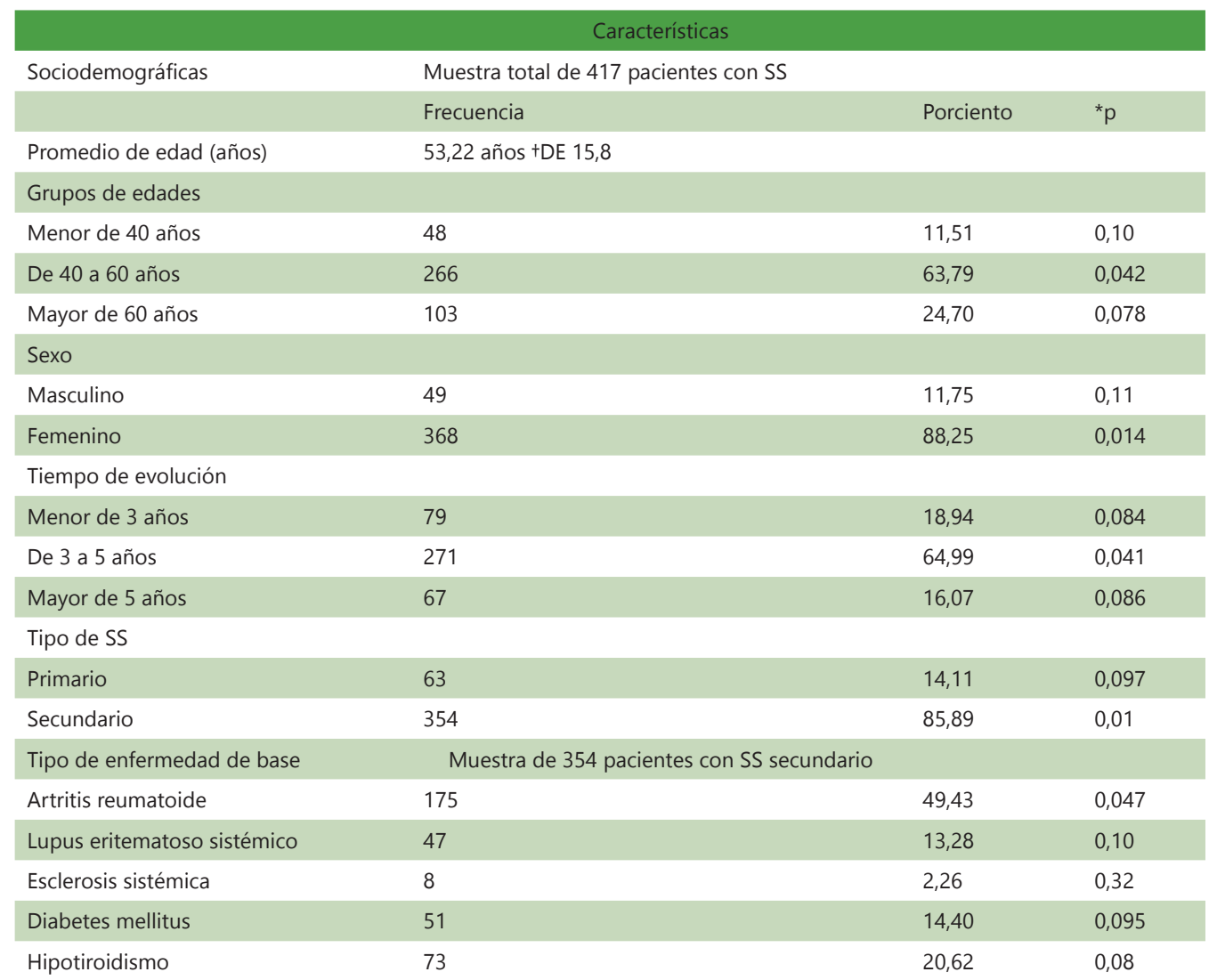

NOTA: Se muestra los resultados del análisis de las caracteristicas generales de los pacientes con SS incluidos en la investigación. Se observa un predominio de pacientes femeninas y de presencia de SS secundario a artritis reumatoide. Fuente: cuestionarios de investigación. $\quad{ }^{2} p=0,05 ; \dagger D E$ : desviación estándar.

Tabla 2. Distribución de pacientes según percepción de calidad de vida global y por dimensiones especificas.

\begin{tabular}{|lllllll|}
\hline Percepción de CVRS & Hombres & Mujeres & Total & & & \\
& Media & ${ }^{*}$ DE & Media & ${ }^{*}$ DE & Media & ${ }^{*}$ DE \\
\hline General & 18,73 & 6,27 & 22,43 & 7,57 & 21,78 & 7,22 \\
\hline Dimensiones & & & & & & \\
\hline Forma física & 3,82 & 1,18 & 3,35 & 0,65 & 3,39 & 1,61 \\
\hline Sentimientos & 3,38 & 0,62 & 3,07 & 0,93 & 3,11 & 0,89 \\
\hline Actividades cotidianas & 4,03 & 0,97 & 3,89 & 1,11 & 3,92 & 1,08 \\
\hline Actividades sociales & 3,11 & 0,89 & 4,03 & 0,97 & 3.99 & 1,01 \\
\hline Cambios en el estado de salud & 3,01 & 0,99 & 2,73 & 1,27 & 2,77 & 1,23 \\
\hline Estado de Salud & 3,57 & 0,43 & 3,11 & 0,89 & 3,17 & 0,83 \\
\hline Dolor & 4,19 & 0,81 & 4,22 & 0,78 & 4,20 & 0,80 \\
\hline
\end{tabular}

NOTA: Se muestra la distribución de pacientes con diagnóstico de SS teniendo en cuenta los resultados de la percepción de CVRS mediante la aplicación del cuestionario de láminas Coop/Wonca. Se observa una peor percepción de CVRS en pacientes femeninas. Fuente: cuestionarios de investigación. *DE: desviación estándar. 
mostraron elevadas puntuaciones fueron la forma física y las actividades cotidianas en los hombres, actividades sociales y cotidianas en las mujeres. Este resultado se puede explicar por el efecto que ejerce el dolor considerado la manifestación más incapacitante del SS, evitando la realización de ejercicios físicos, y características sociodemográficas mostró que mientras mayor fue el tiempo de evolución de la enfermedad, mayor fue la puntuación obtenida del cuestionario de CVRS: a mayor tiempo de evolución de la enfermedad, mayor perpetuación del proceso inflamatorio con más alteraciones inmunológicas y por ende mayor

Tabla 3. Distribución de pacientes según capacidad funcional.

\begin{tabular}{|c|c|c|c|}
\hline \multirow[t]{2}{*}{ Capacidad funcional } & \multicolumn{3}{|l|}{ Muestra total 417 pacientes con SS } \\
\hline & Frecuencia & Por ciento & ${ }^{*} \mathrm{p}$ \\
\hline Sin discapacidad & 159 & 38,13 & 0,069 \\
\hline Con discapacidad & 258 & 61,87 & 0,038 \\
\hline Tipo de Discapacidad & \multicolumn{3}{|l|}{ Muestra 258 pacientes con discapacidad } \\
\hline Discapacidad ligera & 87 & 33,72 & 0,072 \\
\hline Discapacidad Moderada & 133 & 51,55 & 0,043 \\
\hline Discapacidad severa & 38 & 14,72 & 0,097 \\
\hline
\end{tabular}

NOTA: Se muestran los resultados relacionados con la identificación de la capacidad funcional de los pacientes incluidos en el estudio. Destacan como datos significativos el elevado por ciento de pacientes con discapacidad. Se utilizó el cuestionario HAQ-CU. Fuente: cuestionarios de investigación $*_{p}=0$.

Tabla 4. Distribución de pacientes según características sociodemográficas y percepción de CVRS (general).

\begin{tabular}{llc}
\hline & $\begin{array}{l}\text { Características } \\
\text { Sociodemográficas }\end{array}$ & $\begin{array}{l}\text { Muestra total de 417 pacientes con SS } \\
\text { Media }\end{array}$ \\
\hline Tiempo de evolución & & *DE \\
\hline Menor de 3 años & 18,59 & 4,41 \\
\hline De 3 a 5 años & 20,57 & 2,43 \\
\hline Mayor de 5 años & 24,03 & 4,97 \\
\hline Tipo de SS & & 3,87 \\
\hline Primario & 19,13 & 3,14 \\
\hline Secundario & 22,86 & \\
\hline Capacidad funcional & & 2,68 \\
\hline Sin discapacidad & 19,32 & 4,03 \\
\hline Con discapacidad & 22,97 &
\end{tabular}

NOTA: Se realiza el análisis de la percepción de CVRS relacionándola con caracteristicas generales y con la presencia o no de discapacidad; se observa peores percepciones de CVRS en pacientes con SS secundario y en pacientes con discapacidad funcional. Fuente: cuestionarios de investigación *DE: desviación estándar.

provocando así disminución del tono y trofismo muscular, afectando la forma física y la participación en actividades sociales ${ }^{17}$.

El estudio de la capacidad funcional mostró que la mayoría de los pacientes presentan discapacidad moderada, este resultado es similar al encontrado por otros autores al estudiar la capacidad funcional de los pacientes con otras enfermedades reumáticas, aunque son muy escasos los reportes de estudios orientados a la determinación de la capacidad funcional en pacientes con SS 10,12,13.

El análisis bivariado de la percepción de CVRS daño sistémico ${ }^{4,6}$.

Los pacientes con SS secundario también mostraron peor percepción de CVRS, una posible explicación puede estar dada por el efecto de sinergia que tiene la base etiopatogénica del SS y otras enfermedades de base que refuerzan el daño sistémico y aumentan el riesgo de aparición de discapacidad con disminución de percepción de CVRS; esta explicación también es válida para explicar la razón por la que los pacientes con discapacidad presentaron una puntuación mayor del cuestionario de CVRS.

Por último se encontró una fuerte correlación 
positiva entre los valores del cuestionario de capacidad funcional y el de CVRS; de esta relación se interpreta que a medida que aumentan los valores de discapacidad también aumenta la puntuación de percepción de CVRS de los pacientes.

La principal limitación del estudio está relacionada con el sub-registro que existe de pacientes con esta enfermedad en Ecuador. Por otra parte también constituye una limitación los criterios diagnósticos del SS, que incluyen la realización de estudios anatomopatológicos, lo cual desde el punto de vista económico resulta imposible realizar en muchas ocasiones sobre todo para población más vulnerable.

Se concluye que existió una correlación positivafuerteentrelapresenciadediscapacidad y el aumento de los valores de percepción de CVRS, lo que significa que mientras mayor es la discapacidad funcional peor es la percepción de CVRS de los pacientes con SS. Así tambien debido a factores condicionantes como el sexo femenino y el tiempo de evolución mayor de 5 años.

\section{REFERENCIAS}

1.Benítez Falero $Y$, Solis Cartas $U$, de Armas Hernández A, de Armas Hernández Y. Asociación entre Síndrome de Sjögren y enfermedad tumoral: a propósito de un caso. AMC [Internet]. 2016[citado 2019 Oct 11]; 20(2): 219-27. Disponible en:http:// scielo. [ld.cu/scielo.php? script $=$ sci_arttexteIpid $=S 1025$ 02552016000200015 \& Z ing $=e s$

2.Méndez Ramírez D, Murillo Delgado J. Acidosis tubular renal asociado a Síndrome de Sjögren primario. Revista Clínica HSJD [Internet]. 2019 [citado 2019 Oct 06]; 9(5): 45-49. Disponible en: https://revistas. ucr.ac.cr/index.php/clinica/article/view/39642

3. Ladino M, Gasitulli A, Campos X. Sjögren's syndrome: case report. Rev. chil. pediatr. [Internet]. 2015 [citado 2019 Oct 06]; 86(1):47-51. Disponible en:https://scielo.conicyt.cl/scielo.php? script =sci_

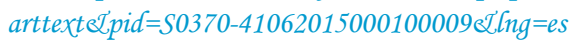

4.de Paiva C, Jones D, Stern M, Bian F, Quianta L, Corbiere S, et al. Altered Mucosal Microbiome Diversity and Disease Severity in Sjögren Syndrome. Scientific Reports [Internet]. 2016 [citado 2019 Oct 11]; 6:23561. Disponible en:https://www.nature.com/ articles/srep 23561

5.Solís Cartas U, Benítez Calero Y, Calvopiña Bejarano SJ, Aguirre Saimeda GL, de Armas Hernández A. Uncommoncombination of hepatocarcinomawith Sjögren's syndrome. Rev Cub Med Mil [Internet]. 2018 [citado 2019 Oct 01]; 47(3):1-9. Disponible en: http:// scielo. sld.cu/scielo.php? script $=$ sci_arttext $\mathrm{Q}$ Z $p i d=S 0138$ 65572018000300012 \&'ing=es

6.Cabrera Escobar D, Ferrer Hurtado O, González Valdés L, Cañadilla González L, Tellería Castellanos AM. Manifestaciones bucales del síndrome de Sjögren. Presentación de un caso. Rev. Med. Electrón. [Internet]. 2016 [citado 2019 Oct 02]; 38(6):877-86. Disponible en: $h t t p: / / s c i e l o . s[d . c u / s c i e l o . p h p$ ? script $=$ sci

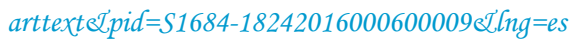

7.Fernández Báez F, Solís Cartas U, Serrano
Espinosa I. Stevens Johnson como complicación de un síndrome de Sjögren. Rev Cuba Reumatol [Internet]. 2016 [citado 2019 Oct 06]: 18(2); 216-218. Disponible en: http://scielo.sld.cu/scielo.php? script $=$ sci_

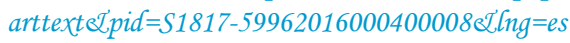

8.Hernández Sampieri R, Fernández Collado C, Baptista Lucio P. (2014). Selección de la muestra. En Metodología de la Investigación (6a ed.). McGrawHill, México; 2014.

9.Solis Cartas U, Calvopiña Bejarano SJ. Comorbilidades y calidad de vida en Osteoartritis. Rev Cuba Reumatol [Internet]. 2018 [citado 2019 Oct 07]; 20(2):e17. Disponible en: http:// scielo. sld.cu/scielo.php? script $=$ sci_arttext\&I $i d=S 1817$ 59962018000200002 \& $\mathcal{L}$ ing $=e s$

10.Prada Hernández DM, Hernández Torres C, Gómez Morejón JA, Gil Armenteros R, Reyes Pineda $Y$, Solís Carta $U$ et al. Evaluación de la calidad de vida relacionada con la salud en pacientes con artritis reumatoide en el Centro de Reumatología. Rev Cuba Reumatol [Internet]. 2015 [citado 2019 Oct 07]; 17(1):48-60. Disponible en:http:// scielo. sld.cu/scielo. php? script $=$ sci_arttexteIpid $=$ S181759962015000100008 \&'ing $=e s$

11.Aguilar J. Experiencia de enfermedad y su asociación con calidad de vida a salud general y oral en mujeres chilenas con Síndrome de Sjögren. Santiago, Chile: Universidad de Chile - Facultad de Odontología [Internet] 2019 [citado: 2019, octubre]. Disponible en: http://repositorio.uchile.cl/6itstream/ handle/2250/168247/Experiencias\%20de\%20enfermedad\%20 en\%20mujeres\%20con\%20sindrome\%20de\%20Sjogren

12.Solis Cartas U, Calvopiña Bejarano SJ, Martínez Larrarte JP, Paguay Moreno ÁR, SaquipayDuchitanga GI. Percepción de calidad de vida en pacientes con osteoartritis. Características sociodemográficas y clínicas. Estudio de $\mathbf{5}$ años. Rev.Colomb. Reumatol. [Internet]. 2018 [citado 2019 Oct 
09]; 25(3): 177-83. Disponible en: http://www. scielo.org.co/scielo.php? script $=$ sci_arttext 2 L $p i d=S 0121$ 81232018000300177 \& $\mathcal{L}$ ing=en

13.Solis Cartas U, Hernández Cuéllar IM, Prada Hernández DM, de Armas Hernández A. Evaluación de la capacidad funcional en pacientes con osteoartritis. Rev Cuba Reumatol [Internet]. 2014 [citado 2019 Oct 07]; 16(1):23-9. Disponible en: http:// scielo. sld.cu/scielo.php? script $=$ sci_arttext 2 L $p i d=S 1817$ 59962014000100004\&'ing=es

14.Ledesma Echevarría C, Vento Pérez RA, León García M, Hernández Rodríguez Y. Nuevos elementos asociados a la fisiopatología de la trombocitopenia inmune primaria en niños. Rev Cubana Hematol Inmunol Hemoter [Internet]. 2017 [citado 2019 Oct 07]; 36 (Suplemento). Disponible en: http://revhematologia.sld.cu/index.php/hih/article/ viewFile/823/637

15.Medina Ortega ÁP, López Valencia D, Mosquera Monje SL, Mora Obando DL, Dueñas Cuéllar RA. Virus de Epstein-Barr y su relación con el desarrollo del cáncer. latreia [Internet]. 2017 [citado 2019 Oct 07]; 30(2):131-45. Disponible en: https://www.redalyc.org/ jatsRepo/1805/180550477003/html/index.html

16.Fernández Martínez G, Zamora Legoff V, Hernández Molina G.Calidad de vida oral en pacientes con síndrome de Sjögren primario. Reumatología Clínica [Internet]. 2020 [citado 2019 Oct 07]; 16(2):926. Disponible en: https://www.sciencedirect.com/science/ article/pii/S1699258X18300913

17.Martínez Larrarte JP, Reyes Pineda Y. Síndrome de Sjögren. Rev cubana med [Internet]. 2010 [citado 2019 Oct 11]; 49(2); 61-76. Disponible en: http://

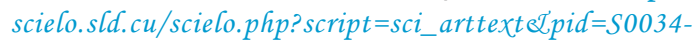
75232010000200006\& 'ing $=e s$

Los autores no refieren conflictos de intereses. 